\title{
Análise epidemiológica dos casos de tuberculose em um município hiperendêmico do Nordeste brasileiro
}

\author{
Epidemiological analysis of tuberculosis cases in a hyper-endemic municipality in \\ Northeastern Brazil
}

\section{Análisis epidemiológico de casos de tuberculosis en un municipio hiperendémico del Noreste de Brasil}

Victor Carneiro de Souza ${ }^{1 *}$, Walter Oliveira Rios Junior ${ }^{1}$, Marcos Vinícius Bezerra Loiola ${ }^{1}$, Yasmin Saboia Moreira ${ }^{1}$, Iana Lia Ponte de Melo ${ }^{1}$, Júlia Oliveira de Assis ${ }^{1}$, Mikaelle Lopes Rodrigues ${ }^{1}$, Luana Maria Moura Ferreira², Gabriel Magalhães Santos², Matheus de Vasconcelos Araújo3.

\section{RESUMO}

Objetivo: Realizar uma análise epidemiológica dos casos de Tuberculose notificados no município de Sobral, no período de 2015 a 2019. Métodos: Trata-se de um estudo descritivo, documental e de caráter quantitativo, realizado por meio da coleta e análise de dados, fornecidos pelo Ministério da Saúde (MS), através do Sistema de Informação de Agravos de Notificação (SINAN) e do Sistema de Informações sobre Mortalidade (SIM). Cada variável foi analisada através de estatística descritiva e do cálculo de indicadores de saúde. Resultados: Foi observado um aumento de $13,7 \%$ na incidência de TB no município, associada uma redução de $51,9 \%$ da taxa de mortalidade. Houve um predomínio de acometimento em indivíduos do sexo masculino $(67,3 \%)$, adultos jovens $(43,7 \%)$, pardos $(79,9 \%)$, com baixa escolaridade $(59,9 \%)$. A taxa de cura foi de $72,1 \%$ e a de abandono do tratamento, $5,3 \%$. Conclusão: 0 município de Sobral, no período analisado, mantém-se com elevado coeficiente de incidência e mortalidade. Além disso, o predomínio da doença em determinados grupos reafirma o fator socioeconômico como um importante contribuinte para sua ocorrência, no entanto, permite o desenvolvimento de estratégias direcionadas que possam levar o município a um melhor cenário epidemiológico.

Palavras-chave: Tuberculose, Epidemiologia, Saúde pública.

\section{ABSTRACT}

Objective: To carry out an epidemiological analysis of Tuberculosis cases notified in the municipality of Sobral, in the period from 2015 to 2019. Methods: This is a descriptive, documentary and quantitative study, carried out through the collection and analysis of data, provided by the Ministry of Health (MS), through the Notifiable Diseases Information System (SINAN) and the Mortality Information System (SIM). Each variable was analyzed using descriptive statistics and the calculation of health indicators. Results: An increase of $13.7 \%$ in the incidence of TB was observed in the municipality, associated with a 51.9\% reduction in the mortality rate. There was a predominance of involvement in males $(67.3 \%)$, young adults $(43.7 \%)$, browns $(79.9 \%)$, with low education $(59.9 \%)$. The cure rate was $72.1 \%$ and the abandonment rate was $5.3 \%$. Conclusion: The municipality of Sobral, in the analyzed period, remains with a high coefficient of incidence and mortality. In addition, the prevalence of the disease in certain groups reaffirms the socioeconomic factor as an important contributor to its occurrence, however, it allows the development of targeted strategies that can lead the municipality to a better epidemiological scenario.

Keywords: Tuberculosis, Epidemiology, Public health.

\section{RESUMEN}

Objetivo: Realizar un análisis epidemiológico de los casos de tuberculosis notificados en el municipio de Sobral, en el período de 2015 a 2019. Métodos: Se trata de un estudio descriptivo, documental y cuantitativo, realizado a través de la recolección y análisis de datos, proporcionados por el Ministerio de Salud (MS), a través del Sistema de Información de Enfermedades Notificables (SINAN) y el Sistema de Información de Mortalidad (SIM). Cada variable se analizó mediante estadística descriptiva y el cálculo de indicadores de salud. Resultados: Se observó un aumento del 13,7\% en la incidencia de TB en el municipio, asociado a una reducción del $51,9 \%$ en la tasa de mortalidad. Predominó la participación en hombres $(67,3 \%)$, adultos jóvenes (43,7\%), marrones $(79,9 \%)$, con baja escolaridad $(59,9 \%)$. La tasa de curación fue del $72,1 \%$ y la tasa de abandono fue del 5,3\%. Conclusión: El municipio de Sobral, en el período analizado, se mantiene con un alto coeficiente de incidencia y mortalidad. Además, la prevalencia de la enfermedad en ciertos grupos reafirma el factor socioeconómico como un importante contribuyente a su ocurrencia, sin embargo, permite el desarrollo de estrategias focalizadas que pueden llevar al municipio a un mejor escenario epidemiológico.

Palabras clave: Tuberculosis, Epidemiología, Salud pública.

1 Universidade Federal do Ceará (UFC), Sobral - CE. *E-mail: victor_cds@yahoo.com.br

2 Universidade Federal do Ceará (UFC), Fortaleza - CE.

3 Universidade Estadual do Ceará (UECE), Fortaleza - CE. 


\section{INTRODUÇÃO}

A tuberculose (TB) é uma doença infectocontagiosa, causada pela bactéria Mycobacterium tuberculosis, também denominada de bacilo de Koch. A transmissão ocorre principalmente através de aerossóis liberados pela tosse, fala ou espirro de um indivíduo com doença ativa. Embora na maioria dos casos a doença acometa os pulmões (forma pulmonar), outros órgãos e sistemas também podem ser afetados (forma extra-pulmonar), principalmente em indivíduos com algum comprometimento imunológico (BRASIL, 2020).

Em 2017, o Ministério da Saúde desenvolveu o "Plano Brasil Livre de Tuberculose", que tem como objetivos reduzir, no país, o coeficiente de incidência da TB em $90 \%$ e o número de mortes pela doença em $95 \%$ até 2035 , em relação aos dados de 2015 . Para o ano de 2020 , o plano tinha uma meta de reduzir em $35 \%$ o número de óbitos e em $20 \%$ o coeficiente de incidência (BRASIL, 2020). A meta é semelhante à estabelecida no "End TB Strategy", desenvolvida pela Organização Mundial de Saúde (OMS), em 2014 (OMS, 2020). Já no Ceará, em 2018, foi desenvolvido o "Plano Estadual de Vigilância e Controle da Tuberculose para o período de 2018-2020" também com o objetivo de reduzir o número de casos e mortes (CEARÁ, 2019).

O colapso nos serviços de saúde provocado pela pandemia de Covid-19, na qual a assistência está mais direcionada aos casos da doença, ameaça o cumprimento das metas preconizadas (OMS, 2020).

Segundo a OMS, em 2019, cerca de 10 milhões de pessoas desenvolveram a doença no mundo e 1,4 milhões morreram devido à tuberculose. Desse total, 208 mil óbitos ocorreram em pessoas HIV-positivas. A organização estimou, ainda, que no mesmo ano, 465 mil casos eram resistentes a medicações utilizadas no tratamento (OMS, 2020).

No Brasil, somente em 2019, 60 mil novos casos foram diagnosticados (BRASIL, 2020). Já no estado do Ceará, de 2008 a 2018, foram notificados quase 40 mil novos casos de TB, com uma média de 3.577 casos por ano, com uma redução de 6,2 \% dos casos ao longo desse período (COSTA NMGB, et al., 2020).

O município de Sobral, no Ceará, tem recebido um destaque negativo em razão da alta incidência de TB, sendo considerado uma região hiperendêmica. Em 2018, foi o município cearense com maior coeficiente de incidência, totalizando 76,5 casos por 100 mil habitantes. Portanto, uma investigação epidemiológica se faz necessária, a fim de identificar fatores e grupos que possam justificar esses resultados, possibilitando 0 desenvolvimento de estratégias direcionadas (CEARÁ, 2019).

O objetivo deste trabalho é realizar uma análise epidemiológica dos casos de Tuberculose notificados no município de Sobral, no período de 2015 a 2019.

\section{MÉTODOS}

Trata-se de um estudo de abordagem descritiva e exploratória, documental e de caráter quantitativo. Foram utilizados dados obtidos no Sistema de Informação de Agravos de Notificação (SINAN), disponibilizados pelo Ministério da Saúde através do Departamento de Informática do Sistema Único de Saúde do Brasil (DATASUS), para a realização de um estudo epidemiológico abordando os casos notificados de Tuberculose no município de Sobral, Ceará, do período de janeiro de 2015 a dezembro de 2019.

Os dados referentes à mortalidade foram coletados através do Sistema de Informações sobre Mortalidade (SIM), utilizando os indicadores do CID-10: A15-A19 (tuberculose). Os dados disponíveis no SIM são coletados com base na causa básica informada pelo médico atestante nas Declarações de Óbitos (DO).

Através da utilização de filtros e cruzamento no programa DATASUS, foram obtidas as seguintes variáveis a fim de traçar o perfil epidemiológico do município neste período: sexo, escolaridade, raça, faixa etária, presença de coinfecção HIV, grupos especiais (gestantes, profissionais de saúde e moradores de rua), presença de comorbidades (diabetes mellitus, Síndrome da Imunodeficiência Adquirida, alcoolismo, 
tabagismo, uso de drogas ilícitas), métodos utilizados para o diagnóstico (baciloscopia, cultura e teste rápido), realização de Tratamento Diretamente Observado (TDO) e desfecho dos casos (óbito, abandono e cura).

Os dados coletados foram tabulados por meio do software Microsoft Office Excel® e, em seguida, analisados através de estatística descritiva, por meio de cálculos de frequência absoluta e relativa. Foram realizados cálculos de média (medida de tendência central), de desvio padrão (medida de dispersão) e de percentis. Os resultados são expressos em porcentagem e/ou como média \pm erro padrão da média.

Por fim, para o cálculo dos coeficientes de incidência e das taxas de mortalidade, foram usadas as projeções anuais para a população, de acordo com o Instituto Brasileiro de Geografia e Estatística, disponíveis para download no site do instituto. Esses indicadores foram determinados para cada ano do estudo, no município de Sobral, no estado do Ceará e no Brasil, a fim de realizar uma análise comparativa dos valores a nível estadual e nacional. Os resultados são expressos em casos (ou óbitos) por 100 mil habitantes (IBGE, 2021).

Em relação aos aspectos éticos, o estudo se adequa à resolução 466/12 do Conselho Nacional de Saúde (CNS). A análise foi fundamentada em bancos de dados de acesso livre, disponíveis na rede mundial de computadores, sendo, portanto, de domínio público. Por essa razão, conforme descreve a resolução, é dispensada a apreciação por um Comitê de Ética e Pesquisa (CEP). Além disso, o estudo zela por seus princípios de não maleficência, beneficência, autonomia e justiça.

\section{RESULTADOS E DISCUSSÃO}

Foi notificado em todo o município de Sobral, no período de 2015 a 2019, um total de 1020 casos, com uma média anual de $204 \pm 17,25$ casos, com uma incidência média anual de 99,3 $\pm 7,2$ casos por 100 mil habitantes. De 2015 a 2019, houve o crescimento de 13,67\% no coeficiente de incidência da doença, aumentando de 95,16 para 108,17 casos por 100 mil habitantes.

Assim, a incidência média anual foi maior que a encontrada no Ceará e no Brasil, no mesmo período (respectivamente 47,12 $\pm 3,3$ e 43,3 $\pm 2,3$ casos por 100 mil habitantes), assim como o coeficiente de incidência identificado em cada ano (Gráfico 1).

Gráfico 1 - Coeficiente de incidência de tuberculose em Sobral-CE, no Ceará e no Brasil, no período de 2015 a 2019 (casos por 100 mil habitantes).

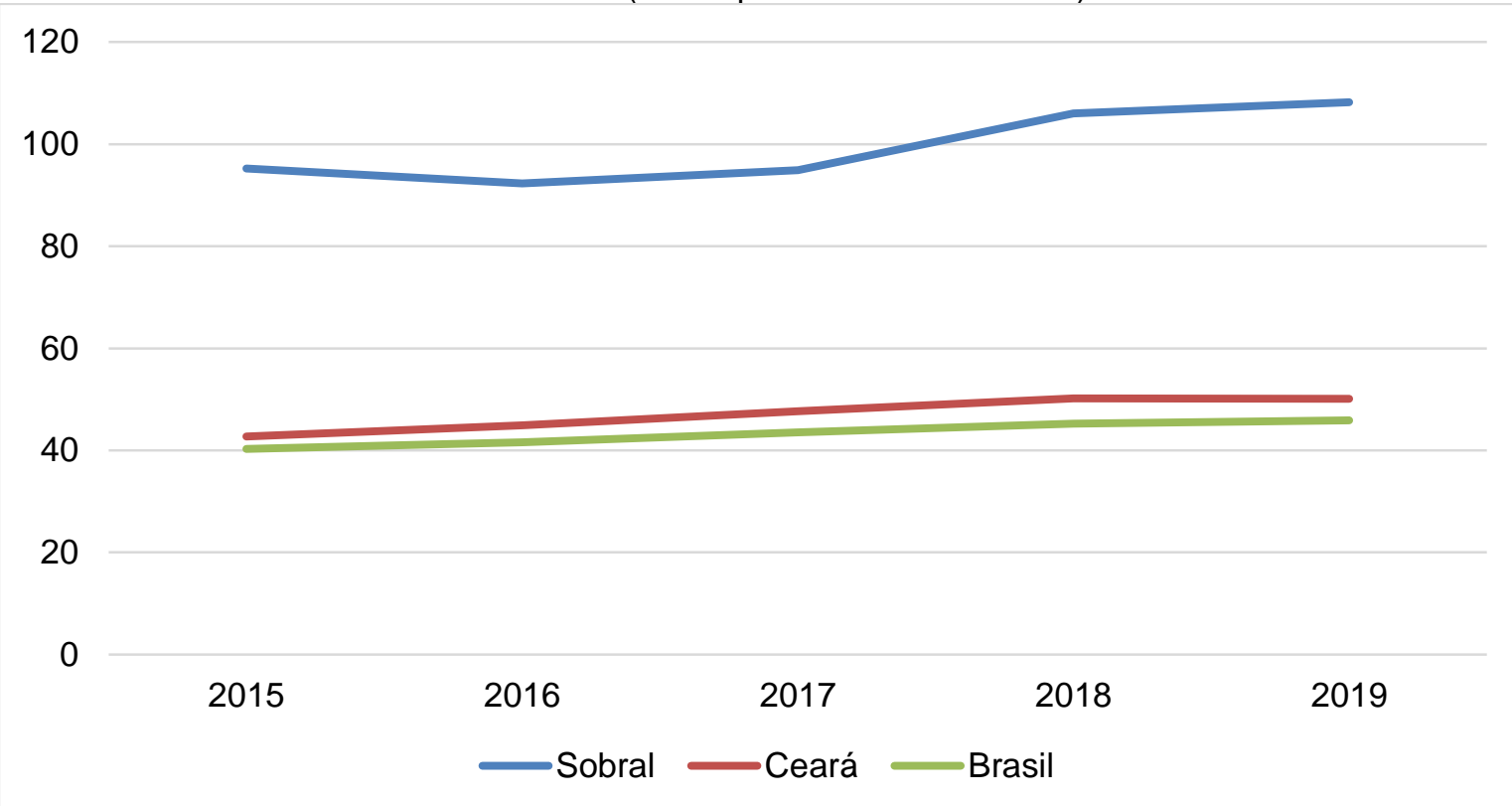

Fonte: Souza VC, et al., 2021; dados extraídos do Sistema de Informação de Agravos de Notificação (SINAN). 
Esse resultado vai em direção contrária às metas estabelecidas no Plano Brasil Livre de Tuberculose pelo Ministério da Saúde, assim como as metas preconizadas pela Organização Mundial da Saúde (OMS), que têm como objetivo a redução do coeficiente de incidência em $20 \%$ até o ano de 2020 , em relação aos dados epidemiológicos do ano de 2015 (BRASIL, 2017).

Em relação à distribuição do número de casos de tuberculose por sexo, em Sobral, foi identificada uma maior incidência em indivíduos do sexo masculino em todos os anos do estudo. Em todo o período analisado, 686 homens (67,25\%) foram acometidos por Tuberculose, com média anual de 137,2 $\pm 12,6$ casos, número bem superior ao encontrado em pacientes do sexo feminino (334 casos, com média anual de $66,8 \pm 9,7$ casos).

Esse resultado acompanha a tendência nacional de predomínio da doença em homens e reforça o que foi observado em outros estudos realizados no país (COSTA NMGB, et al., 2020; BARRETO MTS, et al., 2020; OLIVEIRA AVS, et al., 2019; SOUSA GO, et al., 2020).

O predomínio de casos de tuberculose em homens pode ser justificado pelo fato deste grupo ter um menor cuidado com a própria saúde e buscar menos os serviços de saúde para atendimentos, além de estarem mais expostos aos fatores de risco quando comparados às mulheres (BRANDÃO MGSA, et al., 2018).

Ao avaliar o grau de escolaridade da população acometida pela doença, observa-se que, dentre os 836 casos em que essa classificação foi descrita, 12,2\% dos indivíduos eram analfabetos e 47,7\% possuíam o Ensino Fundamental Incompleto (EFI). Além disso, 8,25\% dos pacientes notificados concluíram o Ensino Fundamental (EFC), 13,35\% tinham Ensino Médio Incompleto (EMI) e 14,59\% Ensino Médio Completo (EMC). Apenas 20 pacientes (2,4\%) cursaram o Ensino Superior (ESI) e $14(1,67 \%)$ já o haviam concluído (ESC).

Observa-se, portanto, que houve predomínio de novos casos de tuberculose em pessoas com baixa escolaridade. Além disso, nota-se que a incidência da doença tende a diminuir conforme o aumento do grau de escolaridade. Os indivíduos com baixa escolaridade também apresentaram maior incidência da doença em outros estudos (BRANDÃO MGSA, et al., 2018; BARRETO MTS, et al., 2020; BARCELOS SSL, et al., 2015; OLIVEIRA AVS, et al., 2019).

A escolaridade é um importante indicador socioeconômico. A baixa escolaridade está intimamente relacionada a baixos níveis de renda, situação que aumenta a vulnerabilidade à tuberculose. A população com baixo nível socioeconômico tem acesso limitado à informação e aos serviços de saúde, assim como está mais sujeita à aglomeração dentro da própria residência, uma vez que geralmente coabitam diversos indivíduos por cômodo (RODRIGUES MW e MELLO AGNC, 2019; SAN PEDRO A e OLIVEIRA RM, 2013). Todos esses fatores podem explicar o predomínio desse grupo na incidência da doença.

Já no que se refere à raça atribuída durante a notificação dos casos, houve importante predomínio de acometimento de indivíduos pardos, com um total de 785 casos e média anual de $157 \pm 9,6$ casos. Tal número corresponde ao percentual de $79,86 \%$ de todos os casos notificados no período. As demais ocorrências foram divididas entre pacientes pretos $(11,09 \%)$, brancos $(7,83 \%)$, amarelos $(1,01 \%)$ e indígenas $(0,2 \%)$.

Resultados semelhantes de predomínio de novos casos de TB em indivíduos pardos também foi observado em outros estudos brasileiros (BARRETO MTS, et al., 2020; BRANDÃO MGSA, et al., 2018).

Ao analisar a idade desses pacientes, houve predomínio de casos em adultos jovens, de 20 a 39 anos de idade. Esse grupo totalizou 446 casos no período analisado, com média anual de 89,2 $\pm 11,6$ casos, correspondendo a $43,73 \%$ dos casos de Tuberculose. A segunda faixa etária mais acometida foi a de 40 a 59 anos (28,33\%), seguida pelo idosos (60 anos ou mais), com $16,08 \%$ dos casos. Em todo o período analisado ocorreram apenas 3 casos $(0,29 \%)$ em crianças menores de 1 ano. Por fim, 118 casos $(11,57 \%)$ ocorrem em crianças e adolescentes ( 1 a 19 anos). No período analisado, houve aumento na incidência em quase todas as faixas etárias (Gráfico 2). 
Gráfico 2 - Número de casos de tuberculose em Sobral-CE, nos anos de 2015 e 2019 (números absolutos).

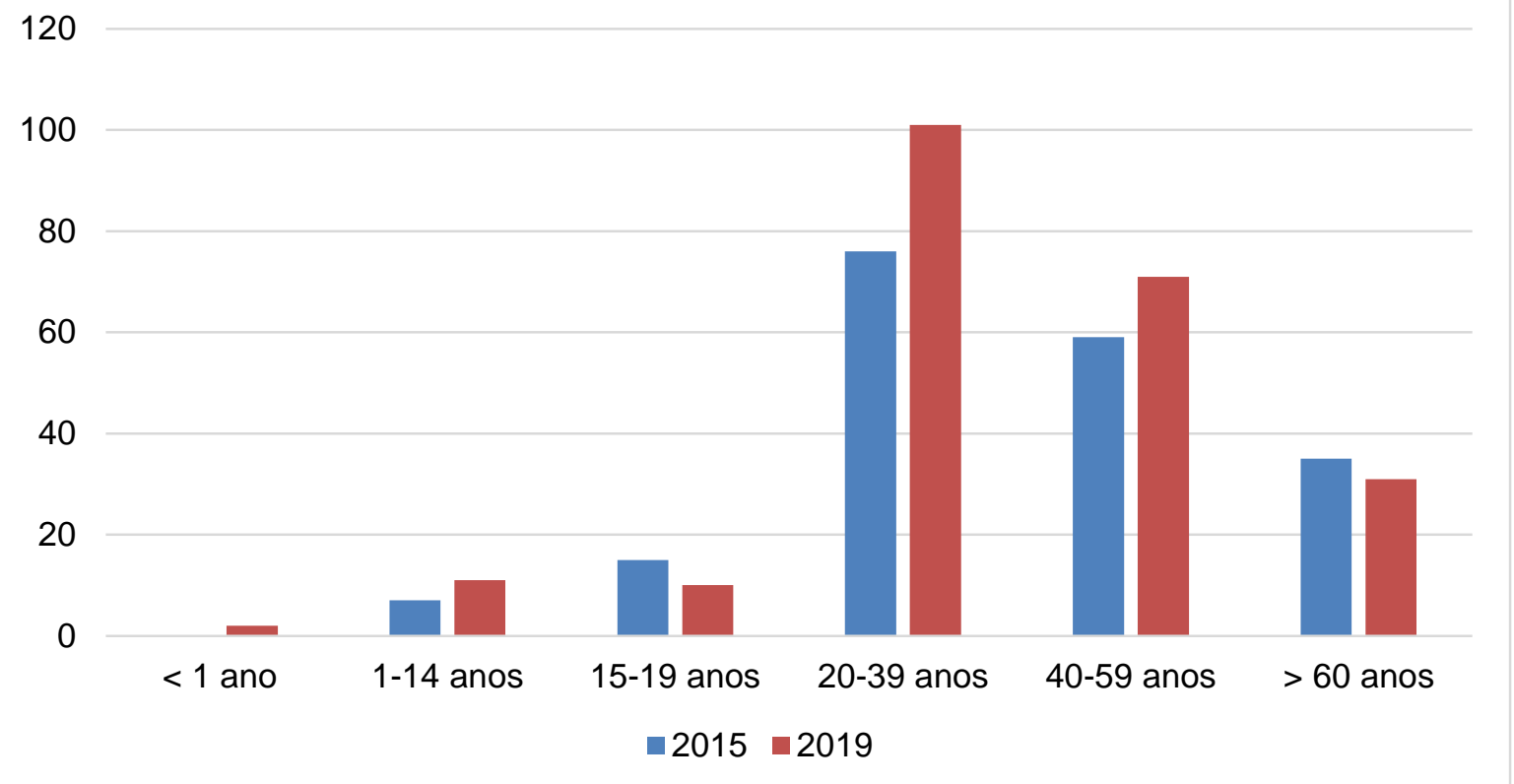

Fonte: Souza VC, et al., 2021; dados extraídos do Sistema de Informação de Agravos de Notificação (SINAN).

Resultados semelhantes foram observados em outros estudos realizados no Brasil (COSTA NMGB, et al., 2020; BARCELOS SSL, et al., 2015; OLIVEIRA AVS, et al., 2019; SOUSA GO, et al., 2020; MACEDO $\mathrm{JL}$, et al., 2017), em que os indivíduos dessa faixa etária foram os mais acometidos. Esse grupo pode ser 0 mais afetado em razão da sua maior exposição a fatores de risco, bem como pelo possível prejuízo financeiro ao núcleo familiar do doente que o adoecimento pode causar (BRANDÃO MGSA, et al., 2018; HINO P, et al., 2011).

Em relação às comorbidades dos indivíduos doentes, 65 (6,37\%) possuíam sorologia positiva para HIV, dos quais, 57 (5,6\%) possuíam diagnóstico de Síndrome da Imunodeficiência Adquirida (SIDA). Além disso, 96 pacientes (9,41\%) eram diabéticos; 243 (23,82\%) eram tabagistas, 178 (17,45\%) eram alcoólatras e 140 $(13,73 \%)$ utilizavam drogas ilícitas.

A infecção pelo HIV é o fator de risco mais importante para a reativação da tuberculose latente, aumentando o risco em 20 vezes. Além disso, a tuberculose é a causa mais comum de óbitos relacionados à SIDA (PAWLOWSKI A, et al., 2012). Desta forma, ambas as doenças agem em sinergismo, debilitando a saúde do indivíduo. Por essa razão, a associação deve sempre ser investigada e medidas devem ser aplicadas para evitar as suas complicações.

Silva DR, et al. (2018) descreve uma importante relação entre diabetes e tuberculose. O baixo controle glicêmico é um fator comumente associado ao desenvolvimento da tuberculose ativa. Esses pacientes têm maior suscetibilidade à infecção em razão de uma redução na função de macrófagos e linfócitos. A associação diabetes e tuberculose está associada a manifestações clínicas e radiológicas mais exuberantes, provavelmente por possuírem uma maior carga bacilar (NASCIMENTO CV e SOARES SM, 2019).

Por outro lado, a tuberculose pode causar uma descompensação do paciente diabético, resultando em sua internação. Sá AMM, et al. (2017) observou em seu estudo, seguindo essa premissa, a internação por complicações do diabetes como uma das causas de abandono do tratamento para tuberculose.

A associação entre tabagismo e tuberculose também é descrita. Os pacientes fumantes podem apresentar disfunção ciliar e uma redução da resposta imune por alterações da atividade dos macrófagos. $A$ taxa de mortalidade por tuberculose é maior em pacientes tabagistas (SILVA DR, et al., 2018). 
Outro aspecto relevante nessas associações é a maior tendência ao abandono do tratamento. Pacientes alcoólatras, fumantes ou usuários de drogas ilícitas apresentam taxas maiores de abandono quando comparado a indivíduos sem esses fatores de risco (SILVA DR, et al., 2018). O uso de bebidas alcoólicas e de drogas ilícitas também foi encontrado como um dos fatores de risco para abandono do tratamento em estudo realizado em Belém, no Pará (SÁ AMM, et al., 2017).

Em todo período foram notificados apenas 4 casos $(0,39 \%)$ em gestantes; 13 casos $(1,27 \%)$ em profissionais de saúde; e $16(1,57 \%)$ em moradores de rua. Os profissionais de saúde, principalmente os da atenção básica à saúde, porta de entrada no atendimento aos pacientes com tuberculose, apresentam uma maior probabilidade de contato com o bacilo e, consequentemente, risco aumentado de infecção. Essa afirmativa é reforçada pelos achados de Lacerda TC, et al. (2017), que observou uma prevalência de $54,1 \%$ de infecção latente pelo Mycobacterium tuberculosis (ILTB) nesse grupo.

Em relação a forma da doença, a grande maioria dos casos notificados eram de tuberculose pulmonar, com um total de 861 e média anual de $172,2 \pm 15$ casos, correspondendo a $84,41 \%$. A forma extrapulmonar estava presente em $13,52 \%$ dos pacientes, enquanto $2,06 \%$ apresentavam ambas as formas.

O pulmão é um local propício para a instalação do Mycobacterium tuberculosis, em razão das altas concentrações de oxigênio (BARCELOS SSL, et al., 2015), o que o torna o sítio mais comum de acometimento da doença, resultado também observado em outros estudos epidemiológicos (COSTA NMGB, et al., 2020; BARCELOS SSL, et al., 2015; OLIVEIRA AVS, et al., 2019; SOUSA GO, et al., 2020).

Em relação à classificação da tuberculose extrapulmonar, os sítios mais acometidos foram a pleura, os gânglios periféricos e os ossos, correspondendo a, respectivamente, 32,08\%, 22,01\% e 8,18\% dos casos de TB extrapulmonar. Além disso, $10,06 \%$ dos casos notificados eram de TB miliar.

Em relação aos métodos diagnósticos, conforme os dados obtidos, a baciloscopia foi realizada em 624 pacientes $(61,2 \%)$, dos quais $525(84,1 \%)$ apresentaram resultado positivo. A cultura foi realizada em 425 pacientes $(41,7 \%)$, dos quais $51,3 \%$ conseguiram isolar o bacilo. Por fim, o teste rápido foi realizado em 458 pacientes $(44,9 \%)$, em que $420(91,7 \%)$ apresentaram resultado positivo, nos quais a resistência à rifampicina foi detectada em $4,05 \%$ das amostras.

Em relação ao desfecho, 735 pacientes (72,06\%) obtiveram a cura e 54 (5,3\%) abandonaram o tratamento. 51 pacientes $(5 \%)$ foram à óbito como complicação da tuberculose, enquanto outros $41(4,02 \%)$ pacientes foram à óbito por outras causas.

Embora apresente uma alta taxa de cura, o município ainda fica aquém da meta de $85 \%$ preconizada pela Organização Mundial da Saúde (OMS). A cura é uma das principais estratégias para redução da morbimortalidade da tuberculose (CEARÁ, 2019). Portanto, é fundamental que o tratamento não seja abandonado e seja realizado de forma correta.

O percentual de abandono também não atingiu a meta preconizada de $5 \%$ estabelecida pela OMS. Os homens, geralmente os mais jovens e solteiros, são os mais vulneráveis ao abandono do tratamento, uma vez que eles tendem a recusar a mudança de hábitos como, por exemplo, cessar o uso de álcool e cigarro, pelo período necessário para o tratamento, de no mínimo 6 meses (SÁ AMM, et al., 2017).

O maior número de abandonos também ocorre em indivíduos com baixa escolaridade. Tal fato pode ser justificado pelo baixo acesso à informação e entendimento sobre a doença e seu tratamento, o que é preocupante, visto que a incidência maior de casos foi observada neste grupo (SÁ AMM, et al., 2017).

Dos pacientes notificados, 846 (82,94\%) realizaram o Tratamento Diretamente Observado (TDO). A TDO consiste na presença de um profissional de saúde ou pessoa capacitada, supervisionando a ingestão do medicamento pelo paciente. Seu objetivo é reduzir a taxa de abandono e, consequentemente, os casos de cepas resistentes (BRASIL, 2019). 
Observa-se, portanto, que um número considerável de pacientes não realizou a TDO, o que pode ter contribuído diretamente para a taxa de abandono do tratamento.

A taxa de mortalidade durante o período avaliado foi variável, com média anual de 2,83 $\pm 1,1$ óbitos por 100 mil habitantes, número discretamente maior que o identificado, no mesmo período, no Ceará (2,36 \pm $0,12)$ e no Brasil $(2,19 \pm 0,04)$. No entanto, foi observada uma redução de $51,9 \%$ na taxa de mortalidade nesse período, caindo de 3,97 para 1,91 óbitos por 100 mil habitantes, se tornando, em 2019, menor que os indicadores no estado do Ceará e do Brasil (Gráfico 3).

Gráfico 3 - Taxa de mortalidade por tuberculose em Sobral-CE, no Ceará e no Brasil, no período de 2015 a 2019 (óbitos por 100 mil habitantes).

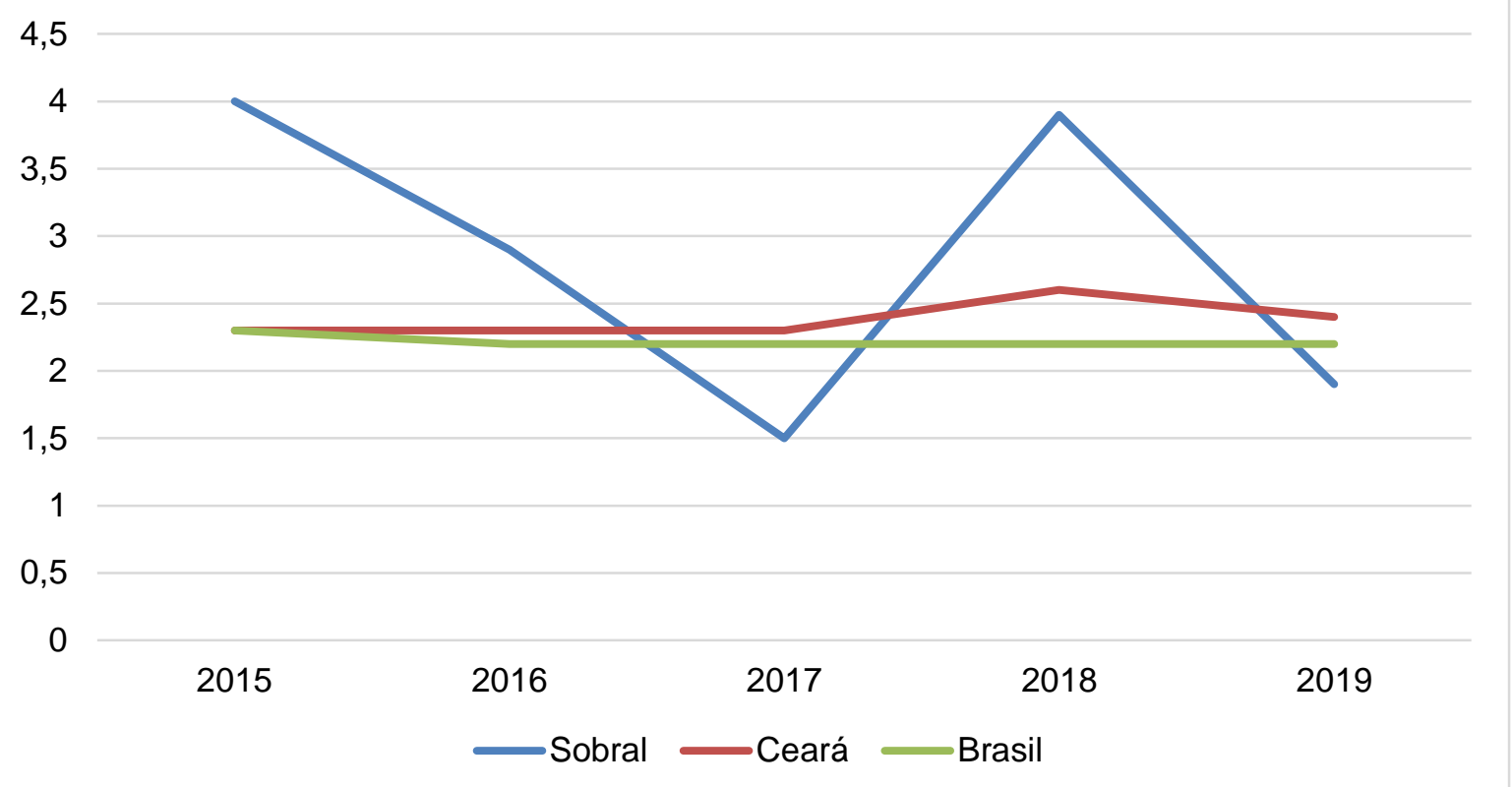

Fonte: Souza VC, et al., 2021; dados extraídos do Sistema de Informações sobre Mortalidade (SIM).

Costa NMGB, et al. (2020) descreve a tendência nacional e mundial de redução das taxas de mortalidade por tuberculose, que pode ser resultado de uma melhora na assistência à saúde, diagnóstico precoce e acompanhamento rígido desses pacientes. Além disso, a meta de $35 \%$ de redução no número de óbitos, estabelecida no Plano Brasil Livre de Tuberculose, foi atingida (BRASIL, 2017).

O predomínio de óbitos ocorreu no sexo masculino, com 18 mortes por tuberculose $(62,07 \%)$. A faixa etária com maior número de óbitos foi a de idosos $(41,38 \%)$, enquanto a raça predominante foi a parda (75,86\%). O maior número de óbitos por TB no sexo masculino e em pardos pode ser justificada por esses grupos serem os mais acometidos pela doença, apresentando, portanto, uma mortalidade numericamente proporcional. O maior número de óbitos em idosos pode ocorrer em razão da comum presença de comorbidades, o que agrava o quadro clínico do paciente infectado e atribui um pior prognóstico.

\section{CONCLUSÃO}

O estudo demonstrou um aumento na incidência e uma redução da taxa de mortalidade por TB no município, que pode ser justificado por uma melhoria da assistência à saúde, com maior número de diagnósticos, tratamento precoce e acompanhamento dos pacientes. O predomínio de acometimento em indivíduos do sexo masculino, adultos jovens, pardos, com baixa escolaridade, assim como na presença de comorbidades e hábitos como tabagismo e etilismo, reafirmam o fator socioeconômico como um importante contribuinte para a ocorrência da doença. Ademais, os resultados são fundamentais para compreensão da distribuição e do comportamento da Tuberculose em Sobral, o que torna possível um reforço e direcionamento das estratégias de saúde em prevenção, diagnóstico precoce e tratamento da doença. 


\section{REFERÊNCIAS}

1. BARCELOS SSL, et al. Análise epidemiológica da Tuberculose no Rio de Janeiro, uma revisão integrativa. Revista Episteme Transversalis, 2015; 9 (2): 61-73.

2. BARRETO MTS, et al. Epidemiologia da tuberculose em um estado do nordeste brasileiro. Research, Society and Development, 2020; 9 (7): e52973643.

3. BRANDÃO MGSA, et al. Avaliação de Indicadores Epidemiológicos da Tuberculose do Município de Sobral, Ceará, 2006 a 2016. Revista UNINGÁ, 2018; 55 (3): 41-51.

4. BRASIL. Ministério da Saúde. Manual de Recomendações para o controle da tuberculose no Brasil. Brasília (DF); 2019.

5. BRASIL. Ministério da Saúde. Plano nacional pelo Fim da tuberculose como Problema de saúde Pública. Brasília (DF); 2017.

6. BRASIL. Ministério da Saúde. Tuberculose. Brasília (DF); 2020. Disponível em: https://www.gov.br/saude/ptbr/assuntos/saude-de-a-a-z-1/t/tuberculose. Acesso em 07 mar. 2021.

7. CEARÁ. Secretaria da Saúde do Estado do Ceará. Boletim epidemiológico: Tuberculose. Fortaleza (CE); 2019.

8. COSTA NMGB, et al. Situação da Tuberculose no Ceará: uma análise epidemiológica. Braz. J. of Develop., 2020; 6 (8): 63049- 63058.

9. HINO P, et al. Perfil dos casos novos de tuberculose notificados em Ribeirão Preto (SP) no período de 2000 a 2006. Ciênc. saúde coletiva, 2011;16 (Suppl 1): 1295-1301.

10. IBGE. INSTITUTO BRASILEIRO DE GEOGRAFIA E ESTATÍSTICA. Projeção de população. Brasil 2021. Disponível em: <https://cidades.ibge.gov.br/brasil/ce/pesquisa>. Acesso em: 13 de mar. de 2021.

11. LACERDA TC, et al. Infecção por tuberculose entre profissionais de saúde da atenção básica. J. bras. pneumol, 2017; 43(6): 416-423.

12. MACEDO JL, et al. Perfil epidemiológico da tuberculose em um Município do Maranhão. ReonFacema, 2017; 3(4):699-705.

13. NASCIMENTO CV, SOARES SM. Manejo integrado de tuberculose e diabetes: uma revisão integrativa. Rev Panam Salud Publica, 2019;43: e21.

14. OLIVEIRA AVS, et al. Perfil epidemiológico da tuberculose no Nordeste do Brasil: série temporal de 2008 a 2018. Research, Society and Development, 2020; 9 (2): e108922129.

15. OMS. ORGANIZAÇÃO MUNDIAL DA SAÚDE. Global Tuberculosis Report. Genebra, 2020.

16. PAWLOWSKI A, et al. Tuberculosis and HIV Co-Infection. PLOS Pathogens, 2012; 8(2): e1002464.

17. RODRIGUES MW e MELLO AGNC. Tuberculose e escolaridade: Uma revisão da literatura. Revista Internacional de Apoyo a la Inclusión, Logopedia, Sociedad y Multiculturalidad, 2018; 4 (2): 1-12.

18. SÁ AMM, et al. Causas de abandono do tratamento entre portadores de tuberculose. Rev Soc Bras Clin Med, 2017;15(3):155-60.

19. SAN PEDRO A, OLIVEIRA RM. Tuberculose e indicadores socioeconômicos: revisão sistemática da litera-tura. Rev Panam Salud Publica, 2013;33(4):294-301.

20. SILVA DR, et al. Fatores de risco para tuberculose: diabetes, tabagismo, álcool e uso de outras drogas. J Bras Pneumol, 2018;44(2):145-152.

21. SOUSA GO, et al. Epidemiologia da tuberculose no nordeste do Brasil. Research, Society and Development, 2020; 9(8): e82985403. 